\title{
Editorial: Enabling High-Penetration Renewable Integration Into a Smarter Grid
}

\author{
Yang $\mathrm{Li}^{{ }^{1 *}}$ and Zhao $\mathrm{Xu}{ }^{2}$ \\ ${ }^{1}$ School of Electrical Engineering, Northeast Electric Power University, Jilin, China, ${ }^{2}$ Department of Electrical Engineering, Hong \\ Kong Polytechnic University, Kowloon, China
}

Keywords: renewable integration, smart grid, uncertainty, renewable forecasting, migration paths

Editorial on the Research Topic

Enabling High-Penetration Renewable Integration Into a Smarter Grid

\section{INTRODUCTION}

With growing concerns on environmental pollution and energy crisis together with the low-carbon transition requirements, renewable energy techniques have been regarded as a crucial solution for ensuring energy clean and sustainable development. Worldwide countries have formulated longterm renewable energy development plans to promote large scale integration of renewable generations into smart grids. However, the variability of renewables will significantly increase the risks and complexities involved in system management and operation during this ongoing energy system transformation. Simultaneously, new emerging techniques in forecasting, operation and optimization provide more regulatory means available for better high-penetration renewable integration (HPRI).

This Special Issue is organized to introduce the latest research in the field of HPRI. At present, there are five papers have been accepted for this Special Issue, which can be sorted into the following three categories including, 1) Review of techniques; 2) Uncertainty modeling of power systems; and 3) Migration paths for smart grids. The three sections below respectively introduce the major researches and contributions of the papers covered in each category.

Specialty section:

This article was submitted to Smart Grids,

a section of the journal Frontiers in Energy Research

Received: 15 October 2020 Accepted: 02 November 2020

Published: 24 November 2020

Citation:

\section{MINI-REVIEWS OF EMERGING TECHNIQUES}

The two obvious disadvantages of renewable energy generations are variability and uncertainty, which increase the difficulty of system reliable operation, and the dynamic operating conditions have a certain impact on electric devices like transformers. Consequently, there are numerous intelligent techniques have been developed in recent years to ensure the economic, safe and reliable operation of power grids and HPRI. Li et al. discussed the basic concepts, principles and applications of six emerging technologies which have significant potentials to promote HPRI in smart grids, including smart inverters, advanced communication protocols, interoperability of renewable devices and systems, peer-to-peer energy trading, renewable forecasting and machine learning, and analyzes the challenges and possible future research directions. Xie et al. comprehensively reviewed the monitoring and protection approaches of different types of transformers in dynamic power 
systems, which is beneficial to maintain secure operation of the power system with high penetration of renewable energies.

\section{UNCERTAINTY MODELING OF RENEWABLE GENERATIONS}

As a highly non-linear random process coupled with multiple variables, renewable energy generation is significantly affected by weather, climate and other meteorological environmental factors. An appreciable increase or decrease of active powers in a short period will cause great harm to the load balance. To better reducing the negative impacts of such unstable power sources in power system planning and operation scheduling, it is necessary to study the power fluctuation characteristics of renewable energies in detail. Wang et al. presented a threeweight mixed Gaussian model to reflect the fluctuation process characteristics of photovoltaic (PV) outputs, and the results compared with other traditional models indicate the proposed method can better fit the fluctuation component. Li et al. proposed an adaptive diffusion kernel density estimation model for PV outputs to accurately describe the uncertainty of PV outputs, besides, a joint probability distribution model of PV output and load is proven to effectively improve the system economy and reduce the bus voltage deviation.

\section{MIGRATION PATHS FOR SMART GRIDS}

Migration paths of smart grids, as a novel concept for meeting the future request of the energy transition, is necessarily considered in planning for network operators. A formal migration path is defined as development steps from one development stage to the next. Flore et al. presented the mutual influence and dependence between different dimensions of migration paths to identify the key development steps, which provides a meaningful reference for the future smart grid planning under HPRI.

\section{CONCLUSION}

The papers in this Special Issue cover different emerging techniques in the forecasting, operation and optimization of the smart grid, which underpin smart grids development with high-penetration renewable energies. The outcome of the research indicates that the application of emerging techniques can promote the ongoing low-carbon transition towards sustainable development.

\section{AUTHOR CONTRIBUTIONS}

All authors listed have made a substantial, direct, and intellectual contribution to the work and approved it for publication.

\section{FUNDING}

This work is partly supported by the "13th Five-Year" Scientific Research Planning Project of Jilin Province Department of Education under Grant No. JJKH20200113KJ.

Conflict of Interest: The authors declare that the research was conducted in the absence of any commercial or financial relationships that could be construed as a potential conflict of interest.

Copyright (c) $2020 \mathrm{Li}$ and Xu. This is an open-access article distributed under the terms of the Creative Commons Attribution License (CC BY). The use, distribution or reproduction in other forums is permitted, provided the original author(s) and the copyright owner(s) are credited and that the original publication in this journal is cited, in accordance with accepted academic practice. No use, distribution or reproduction is permitted which does not comply with these terms. 